\title{
TACKLING AMBLYOPIA IN HUMAN INFANTS
}

\author{
FRANÇOIS VITAL-DURAND ${ }^{1}$ and LOUIS AYZAC ${ }^{2}$ \\ Bron, France
}

\begin{abstract}
SUMMARY
Amblyopia can possibly be avoided if it is detected early and treated appropriately. It remains to be decided whether the general population should be screened or whether a subpopulation of infants likely to develop this pathology can be isolated. A population study using the odds ratio was performed on a group of unselected infants $(n=2143)$ aged 5-15 months who attended a surveillance programme. Results show that a family history of visual defect has a 'protective' effect on individual infants, most probably because more of these infants are brought in for a check-up at an early age on the parents' initiative. The risk factor with the highest predisposition for amblyopia was found to be anisometropia. These results indicate the necessity for screening of refraction and resolution defects in the whole population. The French health authorities have recently decided to provide for a visual assessment visit to all infants at the age of 9 months.
\end{abstract}

Amblyopia is a reduction in visual capacity in one or both eyes that is not improved by refractive correction and occurs in the absence of detectable ocular pathology. Only developmental unilateral amblyopia is considered here. Detailed studies have shown that several aspects of visual function may be affected besides acuity. Usually the reduction in visual acuity, or a reduced contrast sensitivity for high spatial frequencies, is used to diagnose amblyopia on the grounds that it is the visual function most accessible to commonly used tests (for a review see Levi and Carkeet ${ }^{1}$ ). However, it is now clear that the concept of unilateral amblyopia not only affects binocular processes ${ }^{2}$ but also includes deficits of contrast sensitivity, ${ }^{3-5}$ spatial discrimination and skills, ${ }^{6,7}$ visual field and the temporal processing of visual signals affecting motion perception ${ }^{8,9}$ (see

From: ${ }^{1}$ Cerveau et Vision, INSERM Unité 371 and Bébé Vision, Service d'Ophtalmologie, Hôpital Lyon-Sud, France; ${ }^{2}$ Hygiene, Epidémiologie et Information Médicale, Hôpital Lyon-Sud, and Biostatistiques, Informatique médicale, Faculté de Médecine Lyon-Sud, France.

Correspondence to: F. Vital-Durand, Cerveau et Vision, Inserm 371, 18 avenue du doyen Lépine, F-69675 Bron cedex, France. Fax: +33 721315 99. e-mail: vital@lyon151.inserm.fr. review in Tychsen $^{10}$ ) and optokinetic nystagmus. ${ }^{11}$ There is general agreement that amblyopia is often associated with strabismus and impaired binocular function. However, the causal relationship between amblyopia and strabismus is not as clear as it seemed in the past when infants were examined an indefinite time after the onset of strabismus. This is the case also for the presence of a symmetrical optokinetic nystagmus (OKN) which does not constitute an absolute prediction of normal binocular function. ${ }^{12}$

It is thought that an imbalance between the input to the two eyes, implying a decorrelation of signals reaching the cells of the visual cortex, induces a mechanism of binocular competition that causes the signal from one eye to be suppressed. However, there are cases of strabismus which never develop amblyopia, which means that there must be more than one cause of amblyopia.

It is now well accepted that infants are not usually born strabismic although they do not show constant ocular alignment and convergence during the first trimester of life. ${ }^{13}$ In the case of infantile esotropia, amblyopia develops after the onset of strabismus, ${ }^{14}$ but other factors may cause it, among which refractive errors are the most commonly blamed. It is a pathology which occurs in infancy and childhood, possibly up to 5-7 years of age, and the same cause will not lead to this pathology at a later age. It can be cured with an appropriate treatment of patching the sound eye and optical correction within a similar age limit, the treatment being more effective the earlier it is initiated. ${ }^{15}$

Levi ${ }^{16}$ proposes two categories of amblyopia based on their causative origin, although binocular competition is the common mechanism involved: strabismic amblyopia (which implies profound spatial deficits attributable to a coarse grain of cortical spatial sampling) and anisometropic amblyopia (bearing primarily on resolution and contrast sensitivity). In this latter category could be included other optical factors such as excessive hyperopia, severe myopia and against-the-rule astigmatism. However, this distinction is not often accessible in the literature as 
the condition of the patient is described at the age when he or she is studied, the condition prevailing when the amblyopic deficit occurred not being known.

Some deficits are not specific to one category of amblyopes, such as OKN asymmetry. ${ }^{17}$ Rogers et $a l .{ }^{18}$ reported no difference in contrast sensitivity between anisometropic and strabismic amblyopes when patients were matched for visual acuity.

Over the last decade, preferential looking techniques have proved their reliability for measuring visual resolution at an early age. Commercial availability of the Teller acuity cards has contributed to the opening of clinical visual assessment for infants which is progressively expanding. The aim has been to detect strabismus and amblyopia at an early stage corresponding to the peak of the sensitive period the time when therapeutic procedure can be expected to be maximally effective at a minimum cost to the infant's comfort. ${ }^{15}$ Limitations on the availability of early assessment are imposed by the possible lack of responsivity of the infant, visibility of diagnostic signs, compliance with treatment (occlusion and possibly optical correction) and, finally, the parents' attitude toward an unusual medical practice. Another constraint is imposed by cost and health-care policy.

This paper describes an attempt to isolate the population of infants who would benefit from screening and surveillance programmes to prevent amblyopia by evaluating risks factors for amblyopia. A clinical approach to eradicate this pathology, which affects some $3 \%$ of the population in the Western world, is described.

\section{METHODS AND POPULATION}

A visual assessment clinic, called Bébé Vision, was opened in an Ophthalmology Department in Lyon in 1982. Infants were mostly referred by paediatricians or general practitioners; one third were 'walk-ins'. Parents were motivated to take their child to the clinic either because they suspected a visual or ocular problem in their infant (often an epicanthus), or because they were convinced that it was safe to have their infant's eyes examined. Most of the cost of the visit is refunded by the National Health Program (Caisse Nationale d'Assurance Maladie). Data from the first visit of 2413 infants, aged 4-15 months, before any treatment was undertaken, are analysed. There were approximately equal numbers of male and female infants.

It was deliberately advertised that the best age for visual assessment is around 9 months of age. Visits before the age of 7 months were discouraged unless the infant showed signs of strabismus or visual deficit. As a consequence, the age distribution of our population is biased in favour of the range 8-11 months, which constitutes more than half of the total population studied. A typical visit included binocular and monocular acuity measurement with acuity cards, orthoptic assessment, retinoscopy under cycloplegia (tropicamide) and fundus examination. Data were stored on computer. The Epi-Info program was used to process the data to isolate risk factors. Risk factors for amblyopia were analysed using the odds ratios from four categories: pathological cases, nonpathological cases, positive results and negative results.

An odds ratio estimates the probability of a pathology when exposed to a risk factor relative to the probability of pathology when not exposed to that risk factor. Exposure factors considered were family and personal history of general, visual and refractive disorders. The odds ratio quantifies the strength of the association between a factor (e.g. hyperopia) and the occurrence of a pathology (e.g. amblyopia). The greater the amount by which the odds ratio exceeds 1 , the more favourable the situation is for members of that group to develop the pathology. The greater the amount by which the odds ratio is less than 1 , the more the situation is protective for the group. For an odds ratio of 0.5 the probability that the group will develop the pathology is half as great as that for the global population (protective effect). For an odds ratio of 2, the probability that the group will develop the pathology is twice as great as that for the global population (favouring effect).

The refractive errors considered as pathological are somewhat arbitrarily determined in infants, because the level of resolution is limited by retinal rather than optical factors. Values used here (hyperopia when refraction corrected for distance was $\geqslant 3$ dioptres, myopia $\leqslant 0$ dioptre, astigmatism $<-1$ or $>1.5$ dioptres, and anisometropia $\geqslant 1$ dioptre) are traditional. However, we analysed the data using three levels for hyperopia and anisometropia. Similarly, the data concerning amblyopia consider two levels of the difference of resolution between the two eyes: one octave and one half-octave. Infants were not corrected during acuity assessment.

\section{RESULTS}

\section{Incidence of Pathologies}

The largest category of refractive pathology observed is anisometropia, then hyperopia, followed by astigmatism and myopia. Associations between these pathologies are illustrated in Fig. 1. Note that the same distribution is found when a stricter criterion is applied. It is interesting that the observed proportion of infants with either amblyopic or strabismic symptoms is smaller than the proportion of infants with refractive errors defined by even the strictest criterion.

It is clear that there is a higher incidence of 


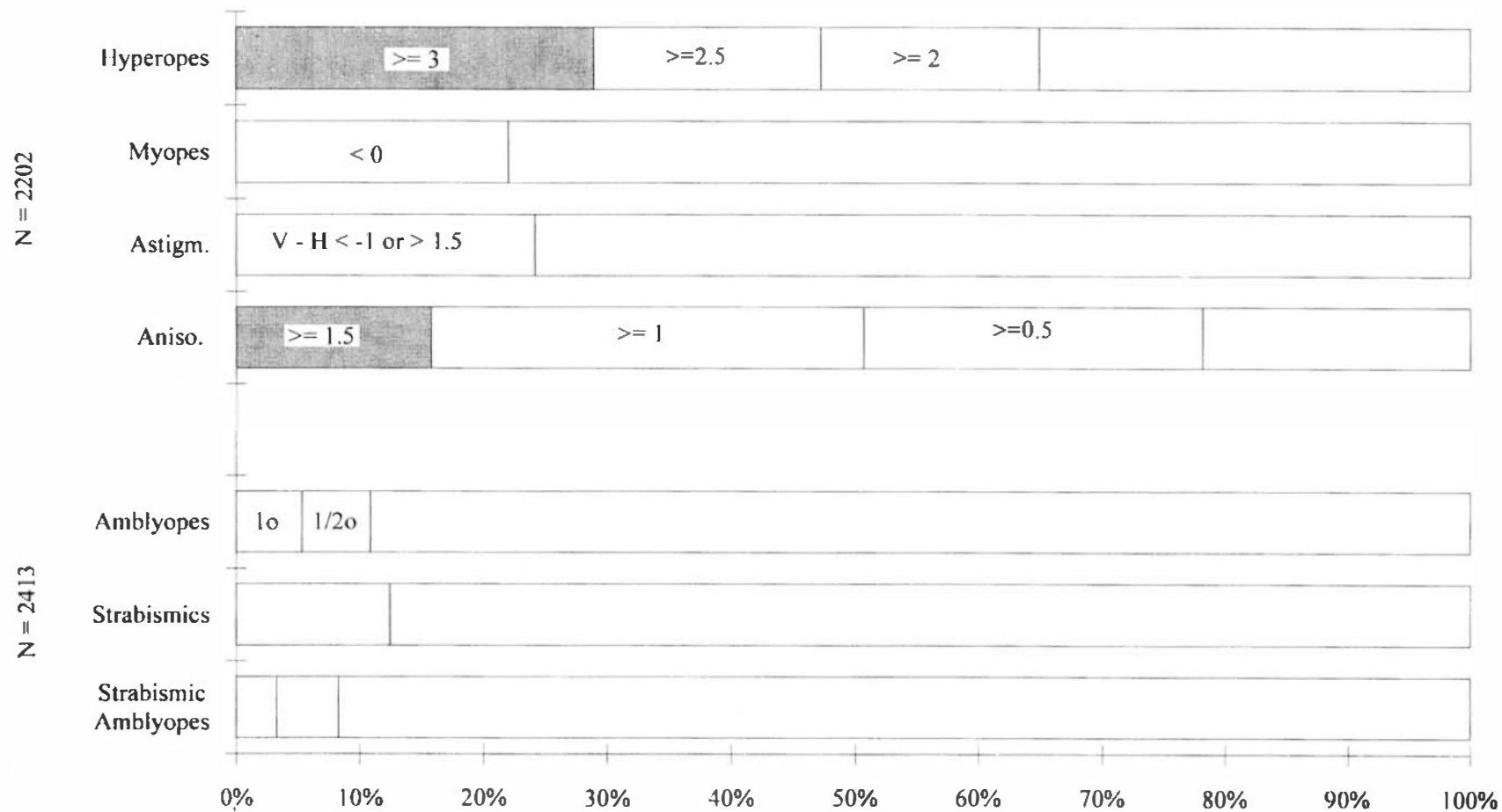

Fig. 1. Incidence of refractive errors and pathologies. Proportion of infants showing each type of refractive error compared with the proportion of infants affected by amblyopia, strabismus, and both. Most infants had more than one type of refractive error. $N$ is the number of infants studied; the number in the lower part of the figure is increased because some infants with amblyopia and/or strabismus were emmetropes.

refractive errors in the population found to be amblyopic in our age range than in the nonamblyopic population, and this difference is highly significant (Pearson KHI2 $=17.66$ ( 5 degrees of freedom), $p=0.0034$; Fig. 2). Nevertheless, $30 \%$ of our amblyopic infants were emmetropes. Conversely only $39 \%$ of non-amblyopic infants were emmetropes. Apart from the smaller proportion of emmetropes in the amblyopic group, the largest difference between the two groups is observed when comparing the group of anisometropic subjects $(17 \%$ versus $7 \%)$.

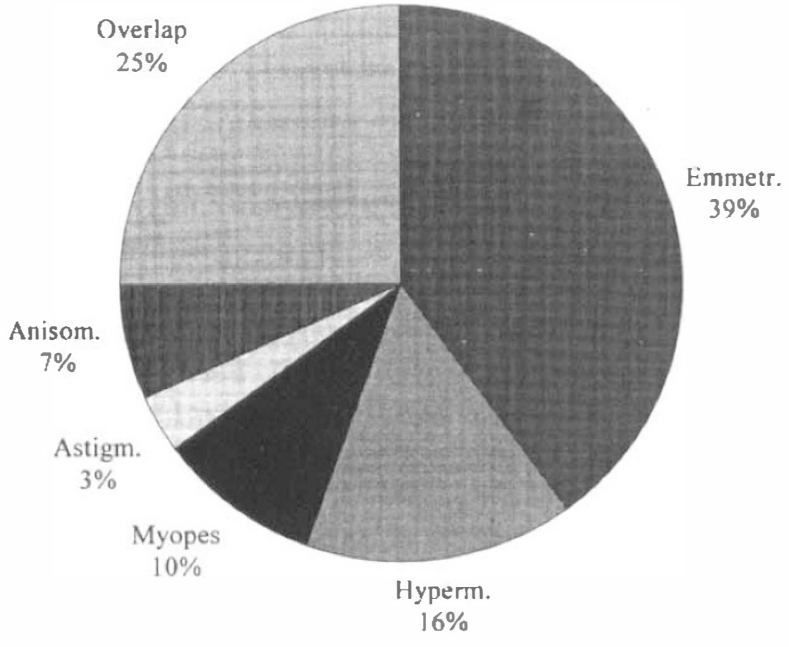

Non-Amblyopic patients

$$
\mathrm{n}=1903
$$

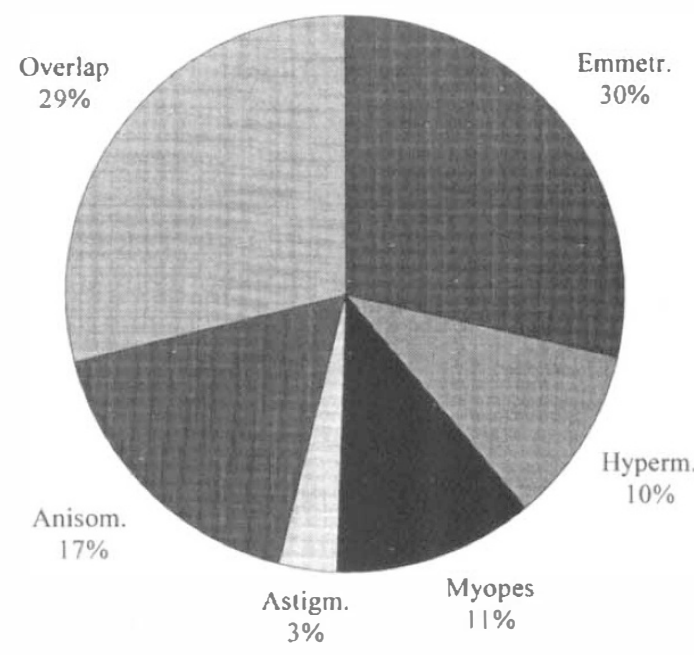

Amblyopic patients

$$
\mathrm{n}=\mathbf{8 7}
$$

Fig. 2. Proportion of refractive errors observed in the group of amblyopic patients compared with the group of nonamblyopic subjects. 'Overlap' refers to infants showing more than one category of refractive error. 


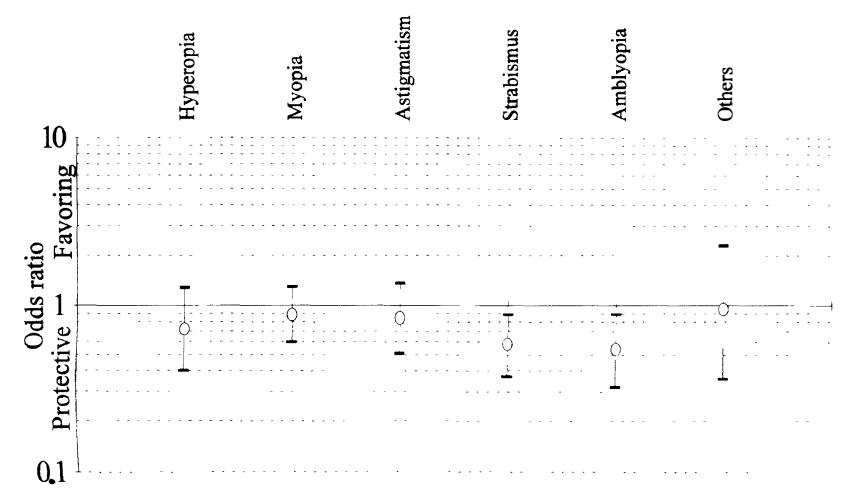

(a)

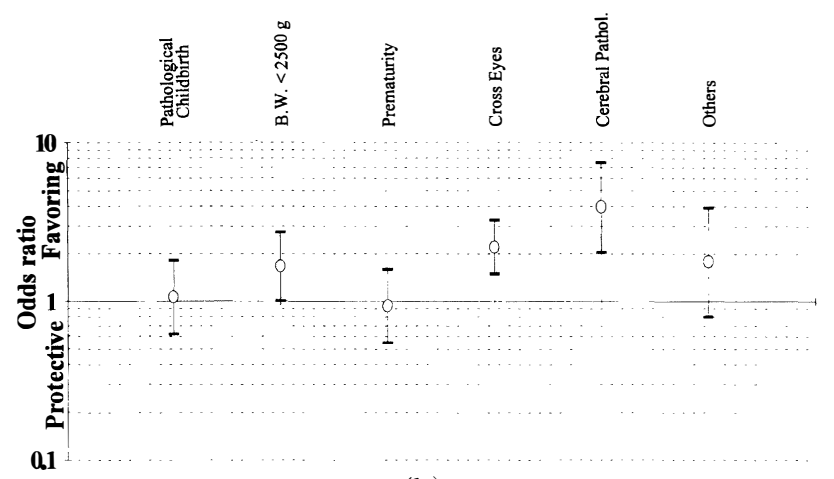

(b)

\section{Risk Factors}

Personal and family risk factors for amblyopia have been tested in the population under study. Fig. 3A shows that a family history of visual problems seems to be protective for the child, possibly through a selection artefact. No pathology reported by the parents has an effect favouring the occurrence of a pathology in the offspring. This protective effect, particularly significant when at least one first-degree relative is strabismic or amblyopic, means that these infants are apparently less prone to develop amblyopia if the parents are affected by these pathologies.

The opposite is observed when personal history is considered (Fig. 3b) and personal refractive condition (Fig. 3c). A report of crossed eyes and cerebral pathology on the one hand and anisometropia on the other are definitely factors favouring the occurrence of amblyopia.

\section{DISCUSSION}

\section{Attendance}

It is probable that our population is biased by several unknown factors. Obviously a large number of parents are worried that their child may have inherited their own visual problem, usually myopia, strabismus or amblyopia. Other infants were systematically referred if they had endured a difficult childbirth or had a birthweight under $1500 \mathrm{~g}$. It could well be that the high proportion of those who attended with a family or personal history reflects

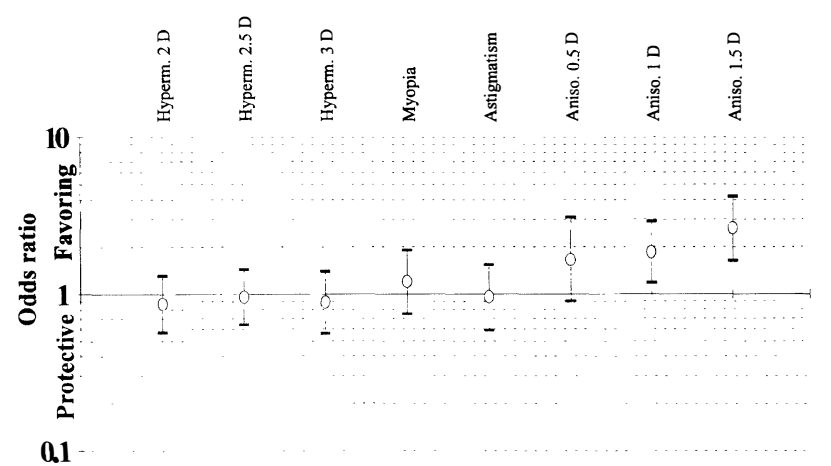

(c)

Fig. 3. Risk factors for amblyopia in the 2113 infants studied for whom the family history was known. In this group there were 124 cases of amblyopia, possibly associated with strabismus. (a) Family history of one of the pathologies quoted. 'Others' refers to any other visual or ophthalmological pathology. (b) Personal history. B.W., birthweight. 'Others' refers to any other pathology (e.g. microphthalmia). (c) Personal refractive error.

some anxiety on the part of the parents. As a consequence the population studied is in no way representative of a general population. In any case it is likely that the bias bears more on the incidence of pathological cases than on the category of pathologies. This is not a limitation of the scope of this study, which is an attempt to identify whether a category of infants can be isolated which would preferentially benefit from an early visual assessment.

\section{Age at Visual Assessment}

In the age range considered, infants around 9 months of age were very responsive to the acuity card procedure; they responded swiftly and complied more easily with orthoptic and ophthalmological examination than at a later age. This choice of age was also justified by the observation that occlusive or optical treatment is more readily accepted by the infant than after the age of 12 months or so. In addition, an occlusion 'dose' of 1 or 2 hours a day is very efficient at this early age in preventing or correcting amblyopia. It is easier for the parents to monitor this short duration of occlusion rather than the longer occlusion regime necessary at any later age. It is also easier to get the infant to accept spectacles, just as items of clothing are accepted, before he or she has constructed a better defined body image.

\section{Risk Factors}

The fact that the infants were not corrected for 
refractive errors when assessed for acuity may have introduced an error in the proportion of amblyopic patients. However, considering the level of resolution at this early age it is not very likely that a refractive error significantly impedes resolution. This is the reason why some authors have questioned the validity of acuity cards as a screening procedure. ${ }^{19}$ Occurrence of amblyopia is not highly correlated with a single symptom or a limited association of symptoms. The same was observed in strabismus. ${ }^{20}$ The single most important factor which emerges from this work is anisometropia. There is an obvious logic behind the fact that anisometropia is causing amblyopia. If the image of one of the two eyes is systematically blurred, especially in the high frequency range, the signals from this eye to the brain are weaker, thus causing a significant imbalance. This can only occur at the age when the infant acquires sensitivity to higher spatial frequencies if infantile anisometropia persists. In this case, optical blurring will cause neural blurring, a reduction in contrast of the image causing a reduction in the signal from that eye. ${ }^{16}$ One may be puzzled by the fact that this factor is the most favourable to amblyopia in this study. It could well be that it results from an unknown bias in our population. However, Flom and Bedell ${ }^{21}$ reported that perhaps one third of their amblyopic patients presented anisometropia but no strabismus. Because, unfortunately, this defect is not detectable without a specific examination, it cannot be used to restrict an appropriate health strategy for the prevention of amblyopia to an 'at-risk' population.

It is confirmed that parental visual pathology has a 'protective' effect on the infants. An obvious hypothesis is that those parents are very aware of the inconvenience caused by their own visual problems and are very likely to have their children examined. Conversely, the population of infants most at risk of developing amblyopia is found in families devoid of visual pathology. In that group, the incidence of amblyopia is low but the probability of it being identified is also very low. Because it is clear that amblyopia also occurs in infants with no parental history of visual defect, a strategy to detect all subjects who might be affected consists of providing visual assessment for the whole population. In conclusion, these data indicate the necessity for screening the whole population for refractive and resolution defects.

This is the new policy of the French national health authorities, who recently took the decision to offer a visual assessment at 9 months of age to all infants. There remains the task of training sufficient specialists to provide this service throughout France. Obviously, each country has different medical surveillance procedures and professional specialisation so that care is provided by various vision specialists. The next stages consist in designing efficient screening procedures and evaluating the cost of screening relative to the benefit of enhanced visual function over the life span.

This work was supported by Inserm, MGEN and Région Rhône-Alpes (contract N 031809301). We are grateful to Andrew James for helping with the English of the manuscript and to the devoted team of Bébé Vision.

Key words: Amblyopia, Early assessment, Infant vision, Risk factors, Screening.

\section{REFERENCES}

1. Levi DM, Carkeet A. Amblyopia: a consequence of abnormal visual development. In: Simons K, editor. Early visual development, normal and abnormal. New York: Oxford University Press, 1993:391-408.

2. Harwerth RS, Levi DM. Psychophysical studies of the binocular processes of amblyopes. Am J Optom Physiol Optics 1983;60:454-63.

3. Gstalder RJ, Green DG. Laser interferometric acuity in amblyopia. J Pediatr Ophthalmol 1971;8:251-6.

4. Hess RF, Campbell FW, Greenhalgh T. On the nature of the neural abnormality in human amblyopia: neural aberrations and neural sensitivity loss. Pflugers Arch 1978;377:201-7.

5. Hess RF, Jenkins S. Amblyopia cannot be explained by considering only detection thresholds. Perception 1980;9:569-76.

6. Fronius M, Sireteanu R. Monocular geometry is selectively distorted in the central visual field of strabismic amblyopes. Invest Ophthalmol Vis Sci 1989;30:2034-44.

7. Fronius M, Sireteanu R. Pointing errors in strabismics: complex patterns of distorted visuomotor coordination. Vision Res 1989;34:689-707.

8. Tychsen L, Lisberger SG. Maldevelopment of visual motion processing in humans who had strabismus with onset in infancy. J Neurosci 1986;6:2495-508.

9. Hamer RD, Norcia AM, Orel-Bixler D, Hoyt CS. Motion VEP in late onset esotropia. Clin Vis Sci 1989;8:55-62.

10. Tychsen L. Motion sensitivity and the origin of infantile strabismus. In: Simons K, editor. Early visual development, normal and abnormal. New York: Oxford University Press, 1993:364-90.

11. Westall CA, Shute RH. OKN asymmetries in orthoptic patients: contributing factors and effect of treatment. Behav Brain Res 1992;49:77-84.

12. Wattam-Bell J, Braddick O, Atkinson J, Day J. Measures of infant binocularity in a group at risk for strabismus. Clin Vis Sci 1987;4:327-36.

13. Hainline L, Riddell PM. Eye alignment and convergence in young infants. In: Vital-Durand F, Atkinson J, Braddick O, editors. Infant vision. Oxford: Oxford University Press, 1996:221-47.

14. Jacobson SG, Mohindra I, Held R. Age of onset of amblyopia in infants with infantile esotropia. Ophthalmol Proc Ser 1980;30:210-6.

15. Vital-Durand F, Patin C. La méthode du regard préférentiel dans une étude longitudinale de 150 enfants de 2 mois à 6 ans. Rev ONO 1991;9:10-1.

16. Levi DM. The Glenn A. Fry Award Lecture. The 'spatial grain' of the amblyopic visual system. Am J Optom Physiol Optics 1988;65:767-86.

17. Roberts N, Westall C. OKN asymmetries in amblyopia: 
their effect on velocity perception. Clin Vis Sci 1990; 5:383-9.

18. Rogers GK, Bremer DL, Leguire LE. The contrast sensitivity function and childhood amblyopia. Am J Ophthalmol 1987;104:64-8.

19. Katz B, Sireteanu R. The Teller Acuity Card test: a useful method for the clinical routine? Clin Vis Sci 1990;5:307-23.
20. Vital-Durand F, Ayzac L, Pinzaru G. Acuity cards and the determination of risk factors in 6-18 months infants. In: Vital-Durand F, Atkinson J, Braddick O, editors. Infant vision. Oxford: Oxford University Press, 1996:185-200.

21. Flom MC, Bedell HE. Identifying amblyopia using associated conditions, acuity and nonacuity features. Am J Optom Physiol Opt 1985;62:153-60. 